\title{
The food's microbiology prediction and quality assessment system based on .NET three-tier architecture
}

\author{
Li-hua JIANG ${ }^{1}$ \\ Dept.of computer \\ Wuhan polytechnic university \\ Wuhan Hubei \\ jianglihua@whpu.edu.cn
}

\author{
Si-gao $\mathrm{LI}^{2}$ \\ Dept.of computer \\ Wuhan polytechnic university \\ Wuhan Hubei \\ Sg51@163.com
}

\begin{abstract}
This paper explores a food's microbiology prediction and quality assessment system platform based on .NET three-tier architecture, which uses ADO.NET caching technology, the MVC architecture, SQL Server database, which establishes fresh food microbiology prediction and quality assessment platform, which includes food news information management, product content management, experiment parameter entry and management, experiment data query and analysis of quality management, the system achieve the research the microbial flora of fresh food in the growth pattern of different temperatures, using the experimental data obtained, establishing a mathematical model, at the same time it selects a better mathematical model as the microbial prediction model of the fresh food by statistical analysis, and eventually making shelf life prediction. Which designs to help the supermarkets systematic management of all types of fresh food, which determinate the supermarket fresh food surface microbial species and its changes.
\end{abstract}

Keywords-ADO.NET;MVC Architecture; SQL Server ; Model Prediction; Quality Assessment

\section{BACKGROUND}

The problem of food quality and safety is very serious all over the world, which is very important to the national economy and the people's livelihood, according to the statistics of the Health Ministry, at present the number of food safety problems and affected area still show a rising trend. Under the existing level of economic development, consumer attitudes and management system, finding appropriate and feasible ways to strengthen the control and management of food safety issues is desperately needed ${ }^{[1]}$. Fresh food is closely related to the majority consumer's life, it mainly refers to the agricultural products in the daily lives of consumers, including vegetables, fruits, fish and meat and so on. Compared to the general food, on the one hand the fresh food is more food safety hazards owing to the sensitivity of the time and environmental conditions etc, on the other hand, it is due to the wide scope food types, a large number of manufacturers, making the fresh food security is difficult to rely solely on the general administrative supervision of the government department.

Supermarket of fresh produce, as a new sale model, the issue of food safety has become a social hot spot, the use of large-scale, integrated business management, good service mode, and a relative improvement food quality assurance system, which occupies the fresh consumer market quickly, buying fresh food through the supermarke has become more and more consumers' choice, excellent fresh food assessment for a supermarket is the fundamental of the "Open Source" ${ }^{[2]}$.

\section{NEED ANALYSIS}

Wherever Times is specified, Times Roman or Times New Roman may be used. If neither is available on your word processor, please use the font closest in appearance to Times. Avoid using bit-mapped fonts if possible. TrueType 1 or Open Type fonts are preferred. Please embed symbol fonts, as well, for math, etc. Fresh food microbiology prediction and quality assessment system is the custom-developed for fresh supermarket food monitoring evaluation software, which helps supermarket systematic management of all types of fresh food, which determinate the supermarket fresh food surface microbial species and its changes, which establishes the microbial growth model, and establishes a rational mathematical model by the experimental data, which forecasts the shelf life of fresh food, to enhance efficiency, avoid mistakes, and get detailed, accurate, real-time the experimental data statistics. System achieve the research about microbial flora distribution of the fresh food in the process of circulation (supermarket) and dominant flora growth rule under different temperatures, to monitor the primary microbial growth prediction by the shelf life of fresh food under different temperatures, using the obtained test data, to establish of a primary, secondary mathematical model, according to statistical analysis selecting the better model as fresh food microorganisms prediction model from the mathematical model, and finally establishing three application software program. Supermarket can easily complete the throughout forecast evaluation process through this software, the front-line staff can track food quality, reduce labor intensity, but also in senior management timely and accurately access a variety of shelf-life information, play a supporting decision-making role. System improves the management decision-making, which reduces business risk and improves the efficiency of food monitoring departments, which provides a scientific and efficient office environment for supermarket, which further improves the comprehensive competitiveness of the fresh food companies ${ }^{[3]}$. 
The system should have the following functions through research and analysis:

- News Trend: News Category dynamic management, the system administrator can add a site related news in the background, foreground real-time show, the viewer can query news according to the type, date, content keyword, the background set the administrator maintenance interface, each news can be edited, set the picture position, graphic layout, hot news, the maintenance interface background administrator can query, modify, delete news by category, date, content rules.

- Safety Monitor: the timely release information of all kinds of safety regulation, visitor can query the detailed real-time information on food safety, the relevant laws and regulations, the Health Ministry food safety notices on the site, collect and acquire all kinds safety monitor information, reasonable layout, according to the various types of experimental test data, which provides more secure monitoring technology and methods to fully protect the scientific and normative data.

- Information Query: which improves the integrative query function about the Web experimental data and testing information, according to various aspects of the fresh food brands, branded products, food category, and microorganisms, as a query condition, query the relevant information.

- Quality Assessment: according to prediction model based on a primary, secondary model driven, which provides any fresh food quality evaluation and analysis biological function, manager and customer at the first time master food quality information, and which provides decision support information for manager, which provides the food quality detailed information for customer.

- Platform Management: which provides the user interface in order to administrator and lab assistant entry the background, which achieves product management, laboratory management, information management, quality assessment, food preservation, general import and export tools: export data to Excel, TXT file from a database, which can also import data to SQL Server database from Excel.

- Experiment Management: which achieves the experimental parameters data modification, entry, save and query function, experimental data in the different temperature entry and modify, the participants of the experiment at any time query the experimental data, which tracks the experimental information to facilitate comparison with the compare.

- Data Query: data query is basically similar foreground function, which achieves all kinds of information inquiry function, convenient for manager and experimenter completing information query and retrieval function. Database connectivity tools can connect database, usually in the first or replace the database.

- Quality Analysis: quality analysis is basically similar foreground function, mainly fresh food quality assessment and analysis capabilities, manager and experimenter in the first time grasp food quality information, which provides decision support information for manager, which provides detailed information about the quality of food for the experimenter, which achieves the remaining shelf life prediction and quality evaluation.

Experiment and platform management is an important part in the fresh food evaluation platform system, which is an important adjunct program of the fresh food assessment, it can also be independent, and it completes fresh food evaluating the whole process ${ }^{[4]}$.

\section{SySTEM THREE-TIER ARCHITECTURE}

The evaluation system design propose is building a high reusability, scalability good code project on the basis of the analysis of project requirement. At present, B / S structure application system based .NET mainly adopts presentation layer, business logic layer and data layer of the three-tier logical architecture, as shown in Fig .1.

The so-called three-tier logical architecture is the UI (User presentation Layer), BLL (Business Logic Layer), DAL(Data Access Layer). UI refers a Web application made up of the client and the browser, which completes the function of the interaction between the user and the system; BLL refers component packaged the business logic of the application, which provides the application interface and data interface as well, which is responsible for the transfer between the user requests and the processing results; but DAL involves database, stored procedure, and database interface components, which provides database call-services from the ADO.NET components to the logical layer ${ }^{[5]}$.

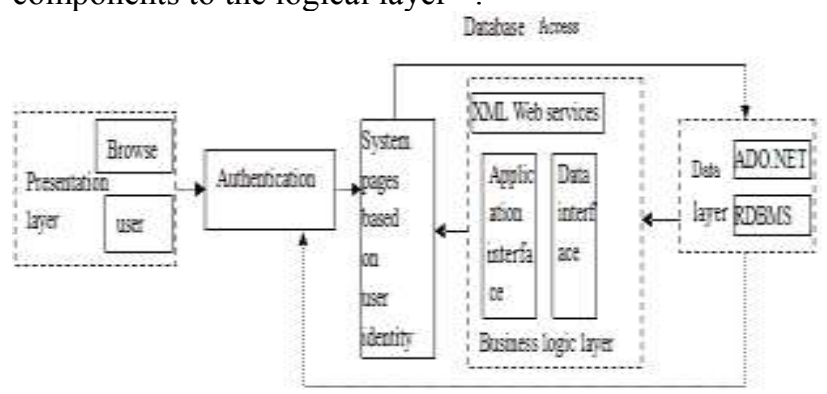

Figure 1. Three-tier Logical Architecture

The user interface of the B / S structure is achieved by the WWW service, very small part of the business logic is achieved in the front-end implementation, which the business logic only is achieved on the server side. This "presentation layer - business logic - data access layer "three-tier architecture is easy to realize different people, from different location, with different access methods to access and manipulate the same data resource, and which can effectively protect data platform and management access power.

.NET technology combines a number of Internet standards including Web services, which simplifies the distributed application development process based on WEB. As an important part of the NET framework, ASP.NET has good scalability and customizability, which 
instant compile and run-caching technology ensure the system has a good response performance.

\section{SySTEM MODULES AND INTERFACE}

Based on the needs analysis combined with the actual situation of the supermarkets, fresh food microbiology prediction and quality evaluation system is designed. The system includes the front and back two parts, the system front aimed at the visitor's client ${ }^{[3]}$, visitors can view news information, safety monitor regulations and policies, while making an inquiry and quality assessment, who accords to the given user and password entering the background management platform management ${ }^{[6]}$.

\section{A. Function Module}

The background is the operating end of the managers completing the fresh food microbiology prediction and assessment in the background, the main background diagram is shown in Fig .2, the backstage management includes news content management, content management, experimental parameters, experimental data entry, experimental data query, quality analysis, the major module functions are described below.

News content management: system site content updates and maintenance, function in the background input, query, modify, delete all kinds of news content, including news and safety monitoring news, press releases in addition to the system administrator does not have news processing privileges, so different users to enter this page which will display different content. Front management page, click the title link into the news reader page, detailed information of each news will be removed, including content, title, keyword, placed in different areas of the page and follow relatively fixed format, the use of all the news substantially the same as the page layout, but the contents of each field corresponding to the type ${ }^{[7]}$.

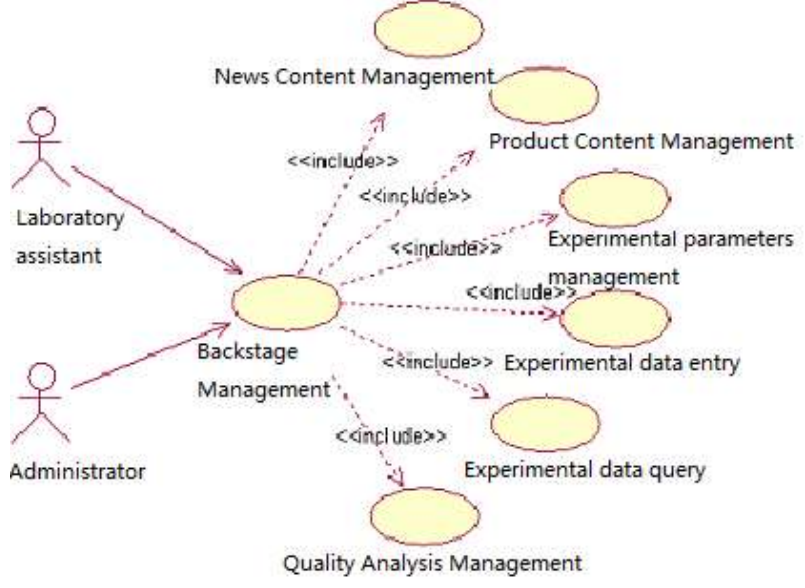

Figure 2. The Main Background Diagram

Product Content Management: management system of fresh food categories, food brands and product names, administrator user can input the food category, food brand and product name, containing the name of the class, parent class, category description, pictures, entry time and other information, information entry is completed, you can click the food category list, a list of food brands and product names list, At this point, each product-related information will be removed, including the category name and category description, the ability to view, edit, delete, etc. The button is provided to an administrator, and relatively fixed in accordance with the format to be placed in a different area of the page for administrators to manage and maintain ${ }^{[8]}$.

Experimental parameter management: System fresh food categories of bacteria, the experimental class, colony characteristics, activity characterized in the type of enzyme, culture conditions, and microscopic characteristics, test indicators, the corruption threshold value management table, the administrator user can input a class of bacteria, experimental category colony characteristics, activity characteristics enzyme type, culture conditions, microscopic characteristics, test index, the corruption threshold table of contents, which contains the species name, product name, category description, the total number of colonies, pictures and input time information entry is completed which you can click the bacterial category list, the experimental category list, a list of colony characteristics, enzyme activity features a list of list of culture conditions, the microscopic characteristics of the list, the list of test indicators, corruption threshold value table, each product-related information will be taken out, containing the name of the category, product name, type description view, edit, delete button, while also providing to the administrator, placed in different areas of the page and follow relatively fixed format to facilitate administrators to manage maintenance.

Experimental parameters entry: fresh food microbiology prediction and assessment, it must first enters the corresponding experimental strain, colony characteristics, microscopic characteristics, activity characteristics of entry, records daily according to the experimental data at different temperatures at different time points the number of colonies, resulting in a certain time period in a temperature growth curve through the SAS statistical software to fit a dynamic model, the growth curve of the experimental temperature, the establishment of the two models.

Experimental data query: fresh food microbiology experiment data integrated query, experimental brand queries, query brand products, food categories and microbial query, the first entering the time information, then which can experiment, units, experiment name, type of food, food brands, branded products, microorganisms name and product barcode SN query, either by a certain kind of information queries, which query can also follow a few of them or more information, clicking the Query button to see a list of detailed experimental information experiment, an experiment name, item barcode $\mathrm{SN}$, the total number of colonies, temperature, humidity, $\mathrm{PH}$ value and the oxygen content is listed in the list of experimental information, and also on a information for viewing, editing, and deletion processing ${ }^{[9]}$.

Quality analysis and management: to predict the number of microbial colonies of fresh food shelf life prediction and risk summary, according to the experimental parameters entry created two models, 
combined with corruption limit the amount of control have been recorded and the largest colony average, fresh food Specifies the initial number of colonies of microorganisms and storage temperature, which can be predicted within a certain period of time, the fresh food specify the change in the number of colonies of microorganisms can be obtained corresponding to the predicted growth curve, level growth forecast; Likewise, in a given different temperatures and initial number of colonies, which can calculate the remaining shelf life, and calculate the relative error of the product risk situations.

\section{B. System Front Main Interface}

Fresh food microbiology prediction and evaluation system front main interface is shown in Fig .3, its main function is to display the Home and business-related information, all kinds of news and regulatory information, user-friendly data query and quality analysis, as well as learn, learning the relevant knowledge of food safety through the platform Management page can enter the admin page.

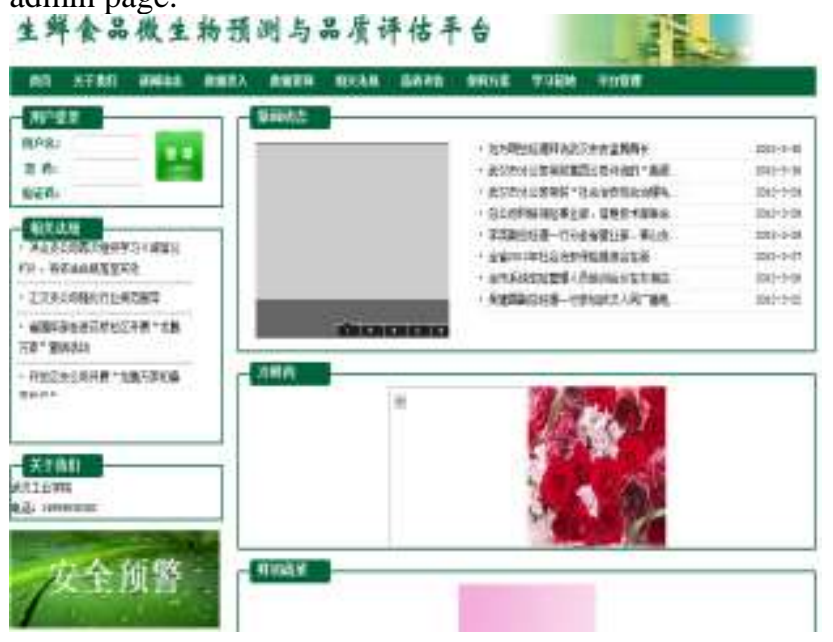

Figure 3. System Front Main Interface

\section{System Background Main Interface}

Prediction and evaluation system of the fresh food microbiology background main interface is shown in Fig .4, its main function is to admin interface system data, only users who have access to enter the management interface, manage news content management, content management of experimental parameters, experimental data entry, data query, the quality of the analysis and management of six modules, mainly for processing of various types of information to add, edit and delete.

The content of the page is part of the code is as follows: public bool ExistsRecord(string name,int parentid)

$\{$ string sql $=$ "select $*$ from a_ppcp_h where name=@name and parentid=@parentid";

OleDbParameter[] pars $=\{$ new OleDbParameter ("@name",name),

new OleDbParameter(" @ parentid",parentid)\};

if (OleDbHelper.ExecuteScalar(CommandType.Text, sql, pars) != null)

return true; else return false; \}

public bool Add(b_cpyz_t para)//记录添加

$\{$ int resu $=0$;

String INSERT_INTO_SQL = "insert into b_cpyz_t (i_sybiaohao,c_symingcheng,f_wendu,f_cpfbyz,c_beizhu1 ,c_beizhu2,c_beizhu3, i_beizhu4, i_beizhu5) values (@i_sybiaohao, @c_symingcheng, @f_wendu, @f_cpfbyz, @c_beizhu 1, @c_beizhu2, @c_bei zhu3, @i_bei zhu4, @i_beizhu5)";

INSERT_INTO_SQL = String.Format (INSERT _INTO_SQL);

OleDbParameter[] pars $=\{$ new OleDbParameter ("@i_sy biaohao", para.I_sybiaohao), new OleDbParameter("@c_symingcheng", para. C_s ymingcheng), new OleDbParameter("@f_wendu",para.F_wendu), new OleDbParameter("@f_cpfbyz",para.F_cpfbyz), new OleDbParameter("@c_beizhu1",para.C_beizhu1), new OleDbParameter("@c_beizhu2",para.C_beizhu2), new OleDbParameter("@c_beizhu3",para.C_beizhu3), new OleDbParameter("@i_beizhu4",para.I_beizhu4), new OleDbParameter("@i_beizhu5",para.I_beizhu5)\}; //将读取出来的温度数据, 放入一个动态数组中去 public ArrayList GetWendu(int symcid) $\{/ /$ 在同一个实验下，温度值可能有重复的，加入 distinct 后值显示唯一的不同值 string sql = "select DISTINCT f_wendu from c_szqx_t where i_sybiaohao=@ symcid"; ArrayList arrs = new ArrayList(); OleDbParameter[] pars $=\{$ new OleDbParameter ("@sym cid",symcid)\}; OleDbDataReader reader $=$ OleDbHelper. ExecuteReader (CommandType.Text, sql, pars); while (reader.Read()) \{arrs.Add(reader["f_wendu"]); $\quad$ return arrs; $\}$

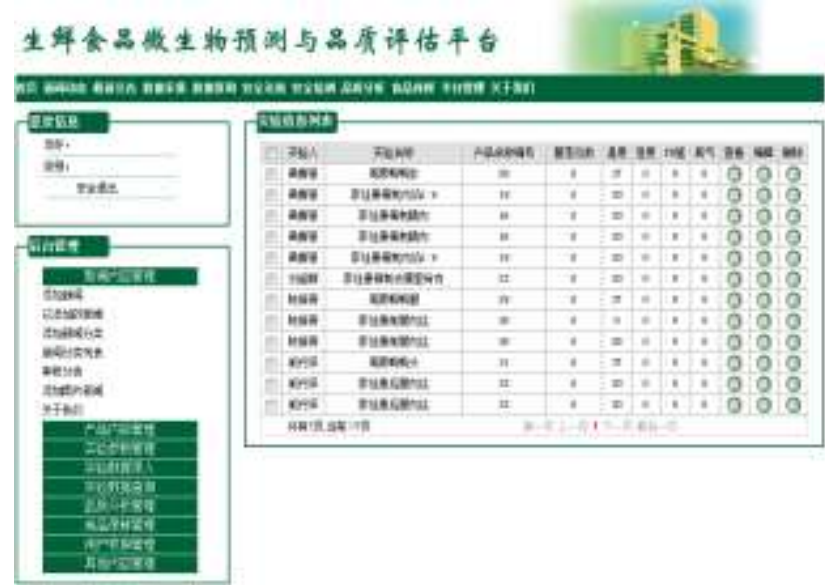

Figure 4. System Background Main Interface

\section{CONCLUSION}

Based .NET three-tier fresh food microbiology forecast and quality assessment system interface is friendly, clear structure, supermarkets managers provide timely and accurate merchandise management, which helps in the supermarket business, management. The system uses a hierarchical design to reduce the dependency between layers, which has good scalability and maintainability, the user only through the logic layer to access the data layer, to reduce the point of entry, to increase the security of the system ${ }^{[10]}$.

The evaluation system successfully tests and analyses (supermarkets) fresh food surface microbial species and its 
variation in the circulation process, microbial growth model, the shelf life of fresh food and the risk of forecast model, which effectively improves the work efficiency and quality of fresh food management, decision-making basis for food policy formulation.

\section{ACKNOWLEDGMENT}

The paper is supported in part by the National Natural Science Foundation of China under Grant No.61201452 and 863Program No.2013AA102302.

\section{REFERENCES}

[1] Li Ju, Wang, Chang Jin Yi, etc. ASP.NET-based office automation system design and implementation [J]. Changshu Institute of Technology, 2011,23 (24) :120-124

[2] Wangliang Ying Based on B/S structure of the building automation system of public colleges and universities [J]. Jilin University: Natural Science,2011,3(6):225-228.

[3] Paul Abraham Building a 3-Tier Application using ASP.NET[EBPOL]. http:// www. c2sharpcorner.Com
[4] Sanjeev Rohilla,Senthil Nathan, Surbhi Malhotral. Microsoft ADO.NET Professional Projects[M].Beijing:China WaterPower Press, 2012

[5] Fay D.An architecture for distributed applications on the internet:Overview of Microsoft's platform[C]//Proceedings of the $17^{\text {th }}$ International Parallel and Distributed Processing Symposium, 2011

[6] Meyer,B.,R. Simon and E.Stapf,Instant .NET Recherche, 2012.67:p.2

[7] ZhangHuiTang,Jamine MacLennan. Data Mining with SQL Server 2010[M], Wiley Publishing,2012,326 329

[8] Scott W.Ambler.The Object Primer 3rd Edition Agile Model Driven Development with UML2[M].New York:Cambrige University Press,2004 Scott W.Ambler.The Object Primer 3rd Edition Agile Model Driven Development with UML2[M].New York:Cambrige University Press, 2012

[9] Robert Ian Mackie.Object oriented implementation of distributed finite element analysis in .NET.UK:ELSEVIER,2011

[10] H.Nishimura,T.Scarvie.Particle Tracking And Simulation on The .NET Framework. USA:EPAC,2012 